\title{
Unclear State of Matter
}

National Cancer Institute

\section{Source}

National Cancer Institute. Unclear State of Matter. NCI Thesaurus. Code C150000.

A state of matter of unknown, unspecified or variable physical characteristics. 